\title{
Parylene Coatings in Medical Devices and Implants: A Review
}

\author{
Sushmitha Kuppusami, Reza H Oskouei \\ School of Computer Science, Engineering and Mathematics, Flinders University, Australia
}

Copyright (c) 2015 Horizon Research Publishing All rights reserved.

\begin{abstract}
This paper reviews various aspects of parylene coatings in medical devices and industry and summarises the fabrication methods of parylene coatings and their potential medical applications. In medical industry, two major beneficial properties of parylene include excellent barrier qualities and inherent bio-compatibility and bio-stability. The need for a bio-compatible material with good surface characteristics is of paramount importance. The recent findings indicate the application of parylene coatings in several areas of medical industry such as surgical instruments, implants, medical devices, mandrels, and medical electronics. Parylene has been slowly introduced into the research market, and has found to be competitive for available materials in the market. A review of the literature was undertaken to identify the prospective use to determine whether parylene coatings can survive the needs in medical industry.
\end{abstract}

Keywords Bio-compatibility, Bio-stability, Surface Characteristics, Parylene, Parylene Coatings, Medical Applications

\section{Introduction}

Developments in medical industry and materials science have significantly changed the technique of replacing any part of human anatomy with a restorative material. Restorative materials are subjected to very threatening environment in which mechanical loads fluctuate rapidly and constantly. Due to the materials, the toxicity of the tissues has increased; thereby, requiring more attention to available materials in the market [1]. Surface coatings deposited onto the materials can protect the surrounding tissues from toxicity, provide good surface properties such as corrosion and wear resistance and also assist the growth of the tissues. Hydroxyapatite (HA) coatings have been widely used in prosthetic applications since they have similar crystallography and chemical composition to the human hard tissues [2]. A strong competent to the HA coatings is parylene which has been used for biomedical applications because of its excellent surface properties, bio-compatibility, bio-stability, increased lubricability and surface consolidation to avert flaking [3]. In 1947, Michael Mojzesz Swarc investigated a class of aliphatic carbon-hydrogen bonds in which the carbon was directly attached to the benzene ring. During the experiments, he heated the simplest compounds at very high temperatures. Para-xylene stood apart from all the chemical substitutes and was first experimented with plastic industry for thermal stability where it proved its mark. Since then, many researchers have used this material in their experiments and in 1965, parylene coatings were commercialised [3].

\section{Parylene Deposition}

The deposition process of parylene is conducted by introducing the polymer in the vapour phase which shows close resemblance as that of the vacuum metallisation. The difference between the two processes is the pressure that is applied. Unlike the vacuum metallisation which has a pressure of $10-15$ Torr, the parylene deposition process employs 0.1 Torr. By this process, all the sides are uniformly impinged by the gaseous monomer [3-5]. This process involves three discrete steps which are shown in Figure 1. The parylene coatings are usually performed at ambient temperatures in the vacuum deposition equipment. The parylene coatings are applied as thin film which grows one molecule at a time as the parylene polymer deposition takes place at the molecular level. A granular raw material called dimer in its solid state is heated under a vacuum and the dimeric gas is generated as a result of vaporisation. The dimeric gas is pyrolised to the monomeric form of the dimer. The monomer gas is deposited as a thin transparent polymer film on the surfaces at room temperature. The advantage of the parylene being applied as gas makes the coatings penetrate the crevices easily and tighten the areas on multi-layer elements with a uniform coating $[6,7]$. The thickness of the coating is based on the applications need. Usually the parylene coatings range in micrometers. They also can vary between hundreds of angstroms to several millimeters [4]. 


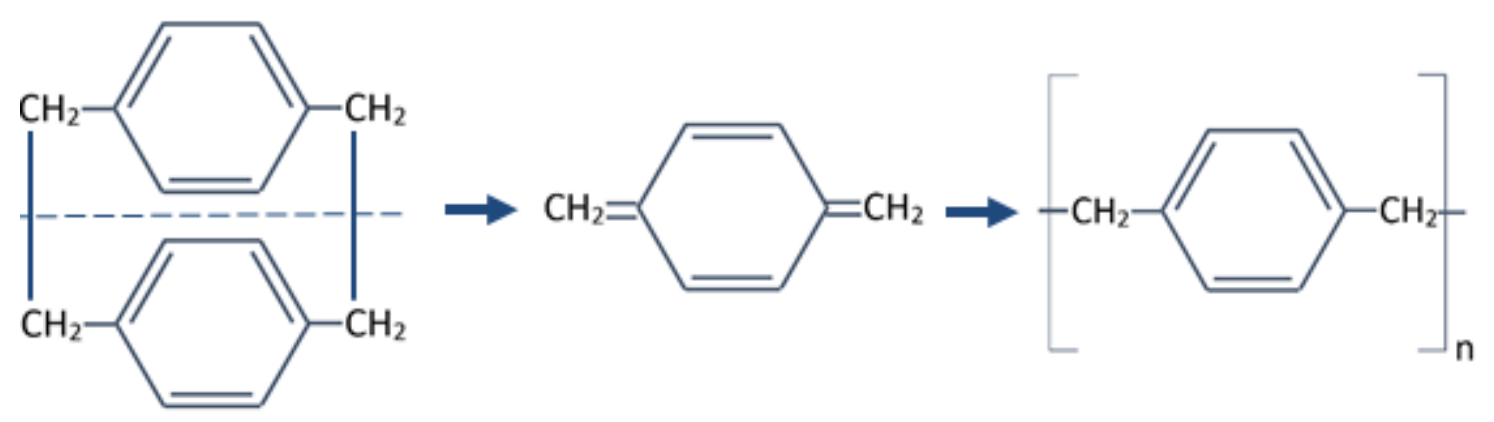

Vaporisation

Pyrolysis

Deposition

Figure 1. The main steps in the parylene coating process.

Table 1. Parylene coatings classifications and their properties.

\begin{tabular}{|c|c|c|c|c|}
\hline & Parylene C & Parylene D & Parylene N & Parylene HT \\
\hline $\begin{array}{l}\text { Structure } \\
{[20]}\end{array}$ & $\begin{array}{l}\text { Completely linear, high } \\
\text { crystalline material, } \\
\text { modified by a substitution of } \\
\text { chlorine atom for one of the } \\
\text { aromatic hydrogen's. }\end{array}$ & $\begin{array}{l}\text { Completely linear, high } \\
\text { crystalline material, modified } \\
\text { by a substitution of chlorine } \\
\text { atom for two of the aromatic } \\
\text { hydrogen's. }\end{array}$ & $\begin{array}{l}\text { Completely linear, high } \\
\text { crystalline material. }\end{array}$ & $\begin{array}{l}\text { Completely linear, high } \\
\text { crystalline material and } \\
\text { replaces the alpha hydrogen } \\
\text { atom of parylene } \mathrm{N} \text { with } \\
\text { fluorine. }\end{array}$ \\
\hline $\begin{array}{c}\text { Aromatic } \\
\text { rings } \\
{[23]}\end{array}$ & {$[\quad]_{n}$} & $L_{\mathrm{Cl}}$ & & $-_{\mathrm{n}}$ \\
\hline $\begin{array}{c}\text { Properties } \\
{[20]}\end{array}$ & $\begin{array}{l}\text { Useful combination of } \\
\text { physical and electrical } \\
\text { properties, low permeability } \\
\text { to moisture and corrosive } \\
\text { gases. }\end{array}$ & $\begin{array}{l}\text { Useful combination of physical } \\
\text { and electrical properties, low } \\
\text { permeability to moisture and } \\
\text { corrosive gases, withstand } \\
\text { slightly higher use } \\
\text { temperatures. }\end{array}$ & $\begin{array}{lrr}\text { Primary } & \text { dielectric, low } \\
\text { dissipation } & \text { factor, high } \\
\text { dielectric strength, low } \\
\text { dielectric constant invariant } \\
\text { with frequency. }\end{array}$ & $\begin{array}{l}\text { Low coefficient friction, } \\
\text { dielectric constant; withstand } \\
\text { high temperature, long term } \\
\text { UV stability and highest } \\
\text { penetrating ability of the four } \\
\text { variants. }\end{array}$ \\
\hline
\end{tabular}

\section{Parylene Types and Applications}

Parylene has been used for more than 30 years and its applications have become wider in several industries. Parylene found its first application as a coating in small ferrite rings in core memory of the electronics. Later, the developments in technology expanded its application to printed circuit boards and many other innovations with the expansion of the electronics industry. As years passed when the industry started its innovation in various fields such as aerospace, military and defence, the use of parylene became wider. In aviation and aerospace, parylene is used for its high altitude and vacuum performance. It is important for the device not to trap air in high altitude as it will not only destroy the device but also can destroy the mission as a whole. Parylene found its success in the aerospace and aviation industry as it trapped no air in coated device or component by its vacuum deposition process [8-13]. In electrical industry, parylene coatings have been widely used as they form a thin layer protecting and stabilising the gentle features with less added mass. Parylene coatings are resistant to high temperature, moisture and hydrocarbons and protect the telecommunication devices without any added mass
[14-19]. From automobile to military and medical industries, parylene has been through a long successful path.

Parylene is unique in its properties which include, pin-hole free coating in thin layers, exact detailing in substrate topography and ability to penetrate through complex substrates. Parylene coatings can be deposited on several substrates such as glass, ceramic, metal, paper, plastic and also on powdered substances. It can be coated on layers as thin as 500 Angstrom [20-22]. Conformal coatings are generally liquid in nature; whereas, parylene coatings are formed on surfaces from a high purity powder known as dimer which has no liquid stage. There are variations of parylene which offer their own coating properties that are slightly different. The commercially available parylene variants include: Parylene C, Parylene D, Parylene N and Parylene HT which are given in Table 1 [4].

\section{Restorative Medical Applications of Parylene Coatings}

The medical industry has expanded itself in the areas of invasive diagnostic and surgical methods. The medical 
implantable device market has grown largely from pacemakers and defibrillators to neuro and spinal stimulating implants. As the size of the medical devices and implants is important, the need for protection has enhanced with the issue of bio-compatibility. To protect the devices and implants from the moisture, bio-fluids and chemicals, a surface coating is required with additional characteristics such as bio-compatibility and bio-stability. Parylene coatings may provide a better durability, bio-compatibility, corrosion resistance, bio-stability, enhanced lubricability, and surface consolidation to avert flaking or dusting [3]; however, it does not provide a good wear resistance which is an important characteristic for a material to be used in medical industry [24]. Hence, larger trials should be implemented to understand the surface properties of parylene to make its stand in the medical industry. In the past decades, the invention of the new variants of parylene such as parylene-HT and parylene-AF4 has become an advantage for the medical device manufactures [25]. Parylene has become an immediate option for manufactures in medical industry. This section gives an insight into the medical applications of parylene coatings.

\subsection{Parylene in Medical Devices}

The need for a bio-compatible coating in the field of implantable medical devices has increased to a greater extent and thus the need for a material which can meet all the requirements for a successful procedure has become very important. Parylene coatings have slowly found their place in the medical devices because of their excellent safety. It has been one of the most preferable materials as it is bio-compatible and does not show any inflammation in the surrounding tissues of the device implanted. Parylene-C is a crystalline, thermoplastic polymer. The deposition process is done using the vapour polymerization technique that ensures polymer films are conformal, uniform and pin-hole free. With all these exceptional properties, parylene becomes a suitable material for all the fabrication devices. The following sections give a few devices where parylene-C has played a vital role and proves its stand as one of the best available coating materials.

Bladder Volume Sensor: Neurogenic bladder is a voiding dysfunction of the central peripheral nervous system such as the diabetes mellitus, cerebrospinal injury or disease or radical pelvic surgery complications [7, 26 and 27]. There are two types of neurogenic bladder, patients with underactive or a contractile neurogenic bladder. The patients with this disorder will not be able to recognize distension of their normal bladder capacity. Hence, the bladder volume sensor which is a real-time monitoring sensor was developed to observe the changes in the bladder volume. When implanting the device in the bladder, it should be coated with a suitable material which is bio-compatible and does not damage the surrounding tissues. The test was performed on different materials and inflammatory cells were observed in the area close to the implants. By H\&E staining, the parylene coating was deposited on the implant and it was observed that there was mild neovascularization close to the implant. Also, the formation of the new blood vessels was not observed in the later stages which makes parylene a suitable coating material for the implant [18]. The Parylene-C coating material has shown safety in the tests carried out in-vitro and in-vivo and has proved to be a safe coating material as it does not show any inflammation on the surrounding tissues of the sensor implanted [28-31].

Electrochemical Impedance Spectroscopy: The encapsulation or the insulation of the device was needed as it would improve the performance of the electrochemical impedance spectroscopy (EIS). The shielding is done to protect the device from harsh, corrosive bio-fluidic environment and also to protect the tissue against the non-biocompatible materials and foreign particles that induce corrosion in the surrounding tissues. The analysis of impedance by experimenting in an appropriate equivalent circuit model showed that the use of sub-micrometer parylene-C coatings has resulted in water diffusion from the top surface very quickly as the film is in contact with solution [32]. Whereas, when coated with a different material, the water is saturated before it reaches the bottom surface. The degree of diffusion depends on the thickness of the coating. A different approach is also available for the encapsulation of the device wherein the top layer of the parylene film can be modified to be more hydrophobic which will make them maintain constant electrical properties at an elevated temperature over the days even when local delamination occurs which can be attained by designing an appropriate device [33-37].

Microelectrode Arrays: The array's used for EMG, endoscopic equipment and other instruments that are in vivo has to be flexible and also bio-compatible. Parylene-C has been used as an alternative to the available coating materials and has shown remarkable results [38, 39]. Parylene is optically transparent through which the tissues can be seen through the array; thereby, it will be easy to position the device. Parylene-C is chemically inert and does not subject to hydrolytic degradation and it has the highest bio-compatibility standards for plastic materials [40].

Saw Devices: The SAW device consists of a piezoelectric substrate with interdigital transducers (IDT's) which works on horizontally polarized surface shear waves (HPSSW) to detect the biomolecules in real time [41]. This substrate is usually coated with lithium tantalate $\mathrm{LiTaO}_{3}$ as it supports both high and low temperature coefficients along with the HPSSW. The binding reactions on this substrate are usually identified using the change in the surface wave velocity which is mainly due to the viscosity and mass adsorption in the sensing layer [42-45]. To increase the sensor characteristics, parylene-C is coated on top of the lithium tantalate as it gives two added features: i) chemically homogeneous substrate and ii) wave guiding layer [41]. Thus, the use of parylene coatings enhances the long-term stability of the implanted device [46]. 


\subsection{Parylene Coatings in Implants}

The need for implants in place of the bone structures has increased to a greater extent in the recent past. Dental implants and orthopaedic implants have been used largely in the medical field. Hence, a successful implant material that is bio-compatible and also stable for long-term is needed. Though finding a suitable implant material was eventually possible, there were factors that arose such as tissue damage and corrosion and wear resistance of the implant due to body fluids and other foreign particles. As a solution to these factors, the implant needs to be coated with a material in which parylene coatings were also found suitable.

Orthopaedic Implants: In Orthopaedic implants, the most commonly used materials are stainless steel and titanium alloy. The parylene $\mathrm{N}$ and parylene $\mathrm{C}$ mechanical and physical properties were examined. Various tests were performed which include micro-indentation, scanning electron and confocal microscopes and scratch tests [24]. Through these tests, it has been proved that the critical load for initial cracks has increased by 3-5 times and the total metal ions release has decreased by 3 times for parylene-C. These properties of parylene-C coating have shown successful results in terms of corrosion resistance but it falls back in wear resistance [47-49].

Dental Implants: In dental implants, the neodymium iron boron magnets coated with parylene-C were used intra orally $[50,51]$. To test the durability of the parylene coated magnet, it was simulated in an oral environment where the parylene coated magnets were subjected to grinding and crushing. It was seen that in few hours, there was visibility of abrasion and wear. Though the parylene-C coating material stands strong in all the surface characteristics and biological properties that suit the body environment, it falls back in the fatigue wear testing [52-55].

\section{Advantages and Disadvantages}

The parylene coating process does not require liquid phase [5]. The main advantage of not including liquid phase is that it does not allow pool in low areas, bridge across substrate or exhibit liquid properties such as meniscus and capillary actions. Parylene having its strength in mechanical properties helps the coating to be achieved in very thin layers [56]. The process of parylene coating results in dry film lubricity which is suitable for many applications and also it can be coated in room temperature. Parylene being coated in very thin layers increases the possibility of protecting very thin substrates without the effect of mechanical loading. One of the most important properties of a material is corrosion and wear resistance [57-59]. Although parylene gives good corrosion resistance, it falls back in the fatigue wear resistance property. Experiments have to be made to find a good alternative solution for parylene as that of the new invention of parylene HT which can withstand high temperature and UV stability. Future studies in parylene should provide a solution for this property and make it one of the best coating materials in medical industry.

\section{Conclusions}

With increasing technological developments in the medical field, the need for a suitable coating material to be used for both in - vitro and in - vivo has become very important [60]. A suitable polymer which has favourable characteristics such as bio-compatibility, bio-stability, enhanced lubricability, high corrosion resistance and wear resistance in order to withstand damage of a device and provide a longer life is needed [61]. This paper gives the basic knowledge of parylene coatings, deposition process, advantages and disadvantages along with the directions on the future research opportunities in the field of biomaterials. Parylene has proven to be a good coating material and is also pin-hole free which makes it easier to coat. It is also a liquid free coating technique; thereby, avoiding disadvantages due to pooling in lower areas. The weak aspects of parylene may include low fatigue and wear resistance. Not all the fields in medicine require this property, but to make parylene- $\mathrm{C}$ a successful coating material, further experiments need to be conducted to improve its fatigue and wear resistance.

\section{Conflict of Interests}

The authors declare that there is no conflict of interests regarding the publication of this paper.

\section{REFERENCES}

[1] Powers JH, ${ }_{\text {th }}$ Sakaguchi RL. Craig's Restorative Dental Materials. 13 ed. Missouri: Elsevier Inc.; 2006. p. 2-4.

[2] Ramli RA, Adnan R, Bakar MA, Masudi SM. Synthesis and characterization of pure nanoporous hydroxyapatite. J PhysSci 2011; 22:25-37.

[3] Parylene Properties Chart, Para Tech Coating, Inc., 2010.

[4] Parylene Properties Chart, SCS Coating, Inc., 2013.

[5] Parylene Specifications \& Benefits Chart, Parylene Engineering, Inc., 2012.

[6] K.G. Pruden, K. Sinclair, S. Beaudoin, Characterization of parylene-N and parylene-C photo oxidation, J. Polym. Sci. A, Polym. Chem. 41 (2003)1486-1496.

[7] Evaluation of the biocompatibility of a coating material for an implantable bladder volume sensor Su-Jin Kim, Dong-Sup Lee, In-Gul Kim, Dong-Wan Sohn, Jung-Yul Park, Bum-Kyoo Choi, Sae-Woong Kim.

[8] Anthony Casasnovas and James W. White., Commercial Plastic Encapsulated Microcircuits for Naval Aviation Applications. 
[9] Simpson J. and C. Boller, 2002: Performance of SMA-reinforced composites in aerodynamic profiles; Proc. Of SPIE Vol.4698, pp.416-426.

[10] Janker P., F. Hermle, T. Lorkowski, S. Storm, M. Wettemann and M. gerle, 2000: Actuator technology based on smart materials for adaptive systems in aerospace; Proc. ICAS 2000, Harrogate, UK.

[11] Kube R. and V.Kloppel, 2001: On the role of prediction tools for adaptive rotor system developments; Smart Master. Struct., 10, pp. 137-144.

[12] O’Regan S.D., B. Burkewitz, C.R. Fuller, S. Lane and M. Johnson, 2002: Payload noise suppression using distributed active vibration absorbers; Proc. Of SPIE Vol. 4698, pp.150-159.

[13] Tennant A. and B. Chambers, 2004: Adaptive radar absorbing structure with PIN diode controlled active frequency selective surface; Smart Master. Struct., 10, pp. 1211-1223.

[14] Tsai-Ning Chen, Dong-Sing Wuu, Chia-Cheng Wu, Cheng-Chung Chiang, Yung-Pei Chen, Ray-Hua Horng., Improvements of Permeation Barrier Coatings Using Encapsulated Parylene Interlayers for Flexible Electronic Applications.

[15] S. Kundu, L. C. Olsen, Thin Solid Films 2005, 471, 298.

[16] M. D. Groner, S. M. George, R. S. McLean, P. F. Carcia, Appl Phys. Lett. 2006, 88, 051907.

[17] T.W. Kim, M. Yan, A.G. Erlat, P.A. McConnelee, M. Pellow, J. Deluca, t.P. Feist, A.R. Duggal, J. Vac. Sci. Technol. A 2005, 23, 971.

[18] D.S. Wuu, T. N. Chen, C. C. Wu, C. C. Chiang, Y. P. Chen, R. H. Horng, F. S. Juang, Chem. Vap. Deposition 2006, 12, 220.

[19] C.C. Tseng, C.W. Chiu, K.X. Zhung, J.H. Lee, G.D.J. Su, Thin Silicon shadow masks for organic light emitting diodes (OLED) deposition process, Proc. SPIE 6414 (2007) 64141G.

[20] Joseph. R. Davis., Handbook of materials for medical devices, pp. 191-192.

[21] L. Wolgemuth, Assessing the Performance and Suitability of Parylene Coating, Med. Dev. Diagn. Ind., Aug 2000, p 42.

[22] W. Leventon, New Coatings and Processes Add Value to Medical Devices, Med. Dev. Diagn. Ind., Aug 2001, p 48.

[23] Materials and Coatings for Medical Devices: Cardiovascular, ASM Materials for Medical Devices Database Committee, $p$. 428-431.

[24] Parylene coatings on stainless steel 316L surface for medical applications - Mechanical and protective properties Monika Cieślik , Marcin Kot , Witold Reczyński, Klas Engvall, Wiesław Rakowski, Andrzej Kotarba.

[25] William R. Dolbier Jr., William F. Beach (July 2003)., Parylene-AF4: a polymer with exceptional dielectric and thermal properties., Journal of Fluorine Chemistry, pp. 97-104.

[26] Samson G, Cardenas DD. Neurogenic bladder in spinal cord injury. Phys Med Rehabil Clin N Am 2007;18:255e74.

[27] Fowler CJ. Neurological disorders of micturition and their treatment. Brain 1999;122:1213e31.
[28] Schmidt EM, McIntosh JS, Bak MJ. Long-term implants of Parylene-C coated microelectrodes. Med Biol Eng Comput 1988;26:96e101.

[29] L. Bowman, J.D. Meindl, The packaging of implantable integrated sensors, IEEE Transactions on Biomedical Engineering 33 (2) (1986) 248-255.

[30] E.M. Schmidt, J.S. McIntosh, M.J. Bak, Long-term implants of parylene-C coated microelectrodes, Medical \& Biological Engineering \& Computing 26 (1988)96-101

[31] J.-M. Hsu, L. Rieth, R.A. Normann, P. Tathireddy, F. Solzbacher, Encapsulation of an integrated neural interface device with parylene C, IEEE Transactions on Biomedical Engineering 56 (1) (2009) 23-29.

[32] Evaluation of sub-micrometer parylene $\mathrm{C}$ films as an insulation layer using electrochemical impedance spectroscopy Wonju Chun, Namsun Chou, Sungbo Cho, Sung Yang, Sohee Kim.

[33] M. Cieslik, K. Engvall, J. Pan, A. Kotarba, Silane-parylene coating for improving corrosion resistance of stainless steel 316L implant material, Corrosion Science53 (2011) 296-301.

[34] R.P. Von Metzen, T. Stieglitz, The effects of annealing on mechanical, chemi-cal, and physical properties and structural stability of parylene C, Biomedical Micro devices 15 (2013) $727-735$.

[35] C. Hassler, R.P. von Metzen, P. Ruther, T. Stieglitz, Characterization of pary-lene $\mathrm{C}$ as an encapsulation material for implanted neural prostheses, Journalof Biomedical Materials Research Part B: Applied Biomaterials 93B (1) (2010)266-274.

[36] X. Xie, L. Rieth, S. Merugu, P. Tathireddy, F. Solzbacher, Plasma-assisted atomic layer deposition of Al2O3and parylene $\mathrm{C}$ bi-layer encapsulation for chronic implantable electronics, Applied Physics Letters 101 (2012) 093702.

[37] E.M. Davis, N.M. Benetatos, W.F. Regnault, K.I. Winey, Y.A. Elabd, The influence of thermal history on structure and water transport in parylene C coatings, Polymer 52 (2011) 53785386.

[38] Flexible parylene-based microelectrode arrays for high resolution EMG recordings in freely moving small animals Cinzia Metallo, Robert D. White, Barry A. Trimmer.

[39] A comparison of the tissue response to chronically implanted Parylene-C-coated and uncoated planar silicon microelectrode arrays in rat cortex Brent D. Winslow, Michael B. Christensen, Wen-Kuo Yang, Florian Solzbacher, Patrick A. Tresco.

[40] http://advancedcoating.com/techinfo/typical.html; Licari, 2003

[41] Chemical modification of parylene C coatings for SAW biosensors Kerstin Lange, Sebastian Grimm, Michael Rapp.

[42] C.A. Flory, R.L. Baer, Surface transverse wave mode analysis and coupling to interdigital transducers, IEEE Ultrason. Symp. Proc. (1987) 313-318.

[43] M.Weiss, W.Welsch, M. von Schickfus, S. Hunklinger, Viscoelastic behaviour of antibody films on a shear horizontal acoustic wave sensor, Anal. Chem. 70 (1998) 2881-2887.

[44] N. Barie, H. Sigrist, M. Rapp, Development of immune 
sensors based on commercially available surface acoustic wave (SAW) devices, Analysis 27 (1999) 622-629.

[45] K. Lange, F. Bender, A. Voigt, H. Gao, M. Rapp, A surface acoustic wave biosensor concept with low flow cell volumes for label-free detection, Anal. Chem. 75 (2003) 5561-5566.

[46] T. Hoshino, I. Saito, R. Kometani, et al., Improvement of neuronal cell adhesiveness on parylene with oxygen plasma treatment, Journal of Bioscience and Bioengineering 113 (2011) 395 .

[47] Silane-parylene coating for improving corrosion resistance of stainless steel 316L implant material Monika Cieslik, Klas Engvall, Jinshan Pan, Andrzej Kotarba.

[48] Oxygen plasma functionalization of parylene $\mathrm{C}$ coating for implants surface: Nanotopography and active sites for drug anchoring M. Gołda, M. Brzychczy-Włoch, M. Faryna, K. Engvall, A. Kotarba.

[49] Bioactive Coatings for Orthopaedic Implants-Recent Trends in Development of Implant Coatings Bill G. X. Zhang, Damian E. Myers, Gordon G. Wallace, Milan Brandt and Peter F. M. Choong.

[50] The durability of Parylene coatings on neodymium - iron boron magnets, Joseph. H. Noar, Asiah Wahab, Robert. D. Evans and Adam. G. Wojcik.

[51] Parylene coating hinders Candida albicans adhesion to silicone elastomers and denture bases resin Lin Zhou, Zhongchun Tong, Guofeng Wu, Zhihong Feng, Shizhu Bai, Yan Dong, Longxing Ni, Yimin Zhao.

[52] Long term effects of orthodontic magnets on human buccal mucosa-a clinical, histological and immunohistochemical study. Lars Bondemark, Juri Kurol and Ake Larrson.

[53] The dual role of Parylene $\mathrm{C}$ in chemical sensing: Acting as an encapsulant and as a sensing membrane for $\mathrm{pH}$ monitoring applications Tatiana Trantidou, David J. Payne, Vasileios Tsiligkiridis, Yu-Chun Chang, Christofer Toumazou, Themistoklis Prodromakis.

[54] J.S. Song, S. Lee, S.H. Jung, G.C. Cha, M.S. Mun, Improved biocompatibility of parylene-C films prepared by chemical vapour deposition and the subsequent plasma treatment, Journal of Applied Polymer Science 112 (2008) 3677.

[55] The effect of Parylene coating on the surface roughness of PMMA after brushing Mariana Santos, Steven Soo, Haralampos Petridis.

[56] D.J. Monk, H.S. Toh, J. Wertz, Oxidative degradation of parylene $\mathrm{C}$ (poly (monochloro-para-xylylene)) thin films on bulk micromachined piezoresistive silicon pressure sensors, Sens. Mater. 9 (1997) 307-319.

[57] Engineering of bone fixation metal implants biointerface-Application of parylene Cas versatile protective coating Monika Cieślik, Sławomir Zimowski, Monika Gołda, Klas Engvall, Jinshan Pan, Wiesław Rakowski, Andrzej Kotarba.

[58] C. Navas, I. Garia, X. Ye, J. de Damborenea, J.P. Celis, Wear 260 (2006) 81 .

[59] Preparation of plasma-polymerized para-xylene as an alternative to parylene coating for biomedical surface modification Chia-Man Chou, Kao-Chang Hsieh, Chi-Jen Chung, Ju-Liang He.

[60] Physicochemical Surface Modification of Materials Used In Medicine - Chapter 1.2.12 Buddy D. Ratner and Allan S. Hoffman p. $254-273$.

[61] Klee D, Hoecker H. Polymers for biomedical applications: improvement of the interface compatibility. Adv Polym Sci 1999;149:1e57. 